\title{
Disponibilidade de informação sobre quantidade de açúcar em alimentos industrializados
}

\author{
Provision of information on the amount of sugar \\ in processed foods
}

Camila Cremonezi Japur (https://orcid.org/0000-0003-0513-1758) 1,4

Dyessa Cardoso Bernardes Assunção (https://orcid.org/0000-0003-4711-7508) ${ }^{2}$

Raíssa Aparecida Borges Batista (https://orcid.org/0000-0002-9928-5872) ${ }^{2}$

Fernanda Rodrigues de Oliveira Penaforte (https://orcid.org/0000-0001-8483-1562) ${ }^{3,4}$

${ }^{1}$ Divisão de Nutrição

e Metabolismo,

Departamento de Ciências

da Saúde, Faculdade de

Medicina de Ribeirão Preto,

Universidade de São Paulo

(USP). Av. Bandeirantes

3900, Monte Alegre. 14049-

900 Ribeirão Preto SP

Brasil.camilajapur@usp.br

${ }^{2}$ Curso de Nutrição,

Faculdade de Medicina,

Universidade Federal de

Uberlândia. Uberlândia

MG Brasil.

${ }^{3}$ Departamento de Nutrição, Instituto de Ciências da Saúde, Universidade Federal do Triângulo Mineiro. Uberaba MG Brasil.

${ }^{4}$ Laboratório de Práticas

e Comportamento

Alimentares, Curso de

Nutrição e Metabolismo,

Faculdade de Medicina

de Ribeirão Preto, USP.

Ribeirão Preto SP Brasil.
Abstract The objective of this study was to assess the provision of information on the amount of sugar and identify the position of sugar in the list of ingredients of processed foods. A cross-sectional study was conducted to analyze all processed traditional and diet/light/zero food products sold in a hypermarket containing the word sugar or sucrose in the list of ingredients. The food labels were read and the position of sugar on the list of ingredients and presence, or absence, of information on the amount of sugar in the nutrition facts table were recorded. Information on the amount of sugar was also requested from the manufacturers by e-mail or telephone. A total of 2,200 food products were assessed, 2,164 (98.4\%) of which were traditional foods and $36(1.6 \%)$ diet/light/zero foods. The amount of sugar was declared in only $14.4 \%$ and $13.9 \%$ of these products, respectively $(p=0.84)$. Only $7.7 \%(n=12)$ of the 156 companies contacted provided the requested information. Sugar was present in the first three positions of the list of ingredients in $75.8 \%$ of the traditional foods and $77.8 \%$ of the diet/light/zero foods ( $p=0.93$ ). The data show that sugar was the main ingredient in the majority of the food products analyzed and that the level of provision of information on the amount of sugar is low.

Key words Sugars, Industrialized Foods, Nutritional Labeling
Resumo $O$ objetivo foi avaliar a disponibilidade da informação sobre quantidade de açúcar e identificar sua posição na lista de ingredientes em alimentos industrializados. Este estudo caracteriza-se como transversal, com análise de todos os alimentos industrializados tradicionais, diet, light e zero comercializados em um hipermercado de grande porte, que contivessem na lista de ingredientes as palavras açúcar ou sacarose. A declaração da quantidade e a posição do açúcar na lista de ingredientes foram registrados após observação do rótulo. A informação de quantidade de açúcar também foi solicitada às indústrias de alimentos, por e-mail ou telefone. Foram analisados 2200 alimentos, sendo $2164(98,4 \%)$ tradicionais e 36 (1,6\%) diet, light e zero, nos quais a declaração da quantidade de açúcar foi observada em apenas $14,4 \%$ e $13,9 \%$ dos alimentos, respectivamente ( $p=0,84)$. Das 156 empresas contatadas, apenas $7,7 \%(n=12)$ disponibilizaram as informações solicitadas. Dentre os alimentos tradicionais, 75,8\% apresentam o açúcar nas três primeiras posições da lista de ingredientes, enquanto nos diet, light e zero, $77,8 \%(p=0,93)$. Conclui-se que o açúcar é o principal ingrediente em grande parte dos alimentos analisados e que a disponibilidade de informação de sua quantidade é baixa.

Palavras-chave Açúcares, Alimentos Industrializados, Rotulagem Nutricional 


\section{Introdução}

Nas últimas décadas ocorreram diversas mudanças alimentares no Brasil e no mundo. Houve aumento do consumo de alimentos com alta densidade energética, ricos em açúcares, gorduras e sódio (ultraprocessados) e redução do consumo de alimentos fonte de fibras e micronutrientes. Tais alterações estão fortemente associadas ao aumento da prevalência de doenças crônicas não transmissíveis (DCNT), como diabetes mellitus, obesidade, hipertensão arterial sistêmica, doenças cardiovasculares e câncer ${ }^{1-6}$.

Evidências epidemiológicas sugerem que o alto consumo de açúcares de adição especificamente é um fator de risco associado às DCNT ${ }^{7}$ ${ }^{11}$. Açúcares de adição (ou livres) referem-se aos açúcares incorporados às bebidas e alimentos na preparação ou processamento industrial ${ }^{9,12}$.

Em 2015, a Organização Mundial de Saúde (OMS) sugeriu a redução do limite máximo diário de ingestão de açúcares de adição de 10\% para 5\% ${ }^{9}$. No ano seguinte, a Organização Pan-Americana de Saúde (OPAS) propôs que alimentos processados e ultraprocessados sejam considerados ricos em açúcares quando a quantidade de açúcares livres for $\geq 10 \%$ do valor energético total (kcal) na porção recomendada ${ }^{13}$. Para que ambas as proposições sejam seguidas, é necessária a disponibilização de informações sobre a quantidade de açúcares presentes nos rótulos de alimentos industrializados.

No Brasil, a declaração do conteúdo energético e de macronutrientes é obrigatória nas tabelas nutricionais de rótulos de alimentos industrializados. No entanto, não há obrigatoriedade de declaração da quantidade de açúcar em alimentos tradicionais, diet, light e zero ${ }^{14,15}$. Essa ausência de informações sobre a quantidade de açúcares gera dificuldades para a escolha alimentar consciente e para o controle da ingestão de açúcar pela população. Portanto, o presente estudo objetivou avaliar a disponibilidade da informação sobre quantidade de açúcar e identificar a posição do açúcar na lista de ingredientes em alimentos tradicionais e diet, light e zero, de sabor doce e salgado, que contêm açúcar.

\section{Métodos}

Trata-se de um estudo transversal no qual foi realizada uma avaliação de alimentos industrializados, tradicionais, diet, light e zero, comercializados em um hipermercado da cidade de
Uberlândia, Minas Gerais, mediante autorização por escrito da gerência do hipermercado. O estabelecimento foi selecionado de forma intencional, pois possui uma grande oferta de produtos industrializados e, de acordo com a Associação Brasileira de Supermercados (ABRAS), é considerada a segunda maior rede de supermercados do Brasil ${ }^{16}$.

A pesquisa foi realizada durante o ano de 2015, em duas etapas. Na primeira foi realizada a observação dos rótulos de todos os alimentos disponíveis no hipermercado avaliado, e a inclusão no estudo de todos os alimentos, de diferentes marcas e sabores, que contivessem as palavras açúcar ou sacarose na lista de ingredientes, independentemente da sua posição. Foram excluídos produtos de mesma composição, marca e sabor, porém com tamanhos de embalagens diferentes. Optou-se por incluir apenas as denominações açúcar e sacarose, e não outros tipos de açúcares (como frutose, glicose líquida, maltodextrina, dextrose, xarope de milho, xarope de frutose, xarope de agrave, xarope de guaraná, lactose, polidextrose, galapolidextrose, maltose, galactose, suco de fruta concentrado, extrato de malte, manitol, xylitol, açúcar invertido, açúcar mascavo, amido, sorbitol, entre outros), por ser este o açúcar de mesa, que é comumente conhecido e utilizado pela população.

Após a seleção, havia o registro do nome técnico, nome fantasia, marca, serviço de atendimento ao consumidor (SAC), posição de sacarose e/ou açúcar na lista de ingredientes; presença ou não da quantidade de açúcar na tabela nutricional.

Na segunda etapa, houve o contato com as indústrias de alimentos, por meio de telefone quando este era gratuito, e-mail ou site do SAC, com o intuito de solicitar informações sobre quantidade de açúcar por porção ou $100 \mathrm{~g}$ do alimento.

Os alimentos selecionados foram separados em dois grupos: Alimentos tradicionais e Alimentos diet, light e zero e classificados em 15 categorias, de acordo com o Sistema Brasileiro de Categorização de Alimentos da Agência Nacional de Vigilância Sanitária (Anvisa) ${ }^{17}$. O termo diet é utilizado em alimentos para fins especiais: com restrição de nutrientes (total ou com quantidades insignificantes), ou naqueles exclusivamente empregados para controle de peso, ou alimentos para dieta de ingestão controlada de açúcares ${ }^{15}$. O termo light é atribuído no rótulo como uma informação nutricional complementar, e seu uso é permitido quando há redução de algum nutriente em pelo menos $25 \%$ comparado ao tra- 
dicional, ou quando seu conteúdo absoluto respeita limites do atributo "baixo"; e o termo zero, quando de acordo com o atributo "não contém", estabelecidos na RDC 54/2012 ${ }^{18}$.

Posteriormente à coleta e digitação dos dados, as informações foram revisadas por outros dois pesquisadores e executou-se análise estatística descritiva do número e da porcentagem dos resultados obtidos. A comparação dos percentuais de declaração da quantidade de açúcares e da presença do açúcar nas três primeiras posições da lista de ingredientes, entre alimentos tradicionais e diet, light e zero, foi feita pelo teste qui-quadrado, utilizando-se nível de significância de 5\%. A análise estatística foi realizada no software GraphPad Instat versão 3.05.

\section{Resultados}

Foram avaliados 2.200 alimentos industrializados que continham na lista de ingredientes, independentemente de sua posição, a palavra açúcar ou sacarose. Do total de alimentos avaliados, $98 \%(\mathrm{n}=2164)$ eram tradicionais e $1,6 \%$ eram diet, light e zero $(\mathrm{n}=36)$.

A informação quantitativa sobre o açúcar estava presente nos rótulos de 14,4\% dos alimentos tradicionais e $13,9 \%$ dos diet, light e zero, sem diferença entre os grupos $(p=0,84)$. A declaração de açúcar nos alimentos tradicionais foi encontrada principalmente nos grupos: Balas, doces, bombons e confeitos (35,3\%); Preparados para adicionar ao leite (26,5\%); Cereais e/ou produtos à base de cereais (22,9\%); Petiscos $(20,4 \%) \mathrm{e}$ Produtos de panificação e Biscoitos (19,8\%). Nos alimentos diet, light e zero, a declaração foi encontrada somente nos grupos: Preparados para adicionar ao leite $(50,0 \%)$ e Produtos de panificação e biscoitos $(30,0 \%)$, conforme descrito na Tabela 1.

O açúcar estava presente nas 3 primeiras posições da lista de ingredientes em 1.668 (75,8\%) alimentos industrializados, que corresponde a $75,8 \%$ dos tradicionais e $77,8 \%$ dos diet, light e zero $(\mathrm{p}=0,93)$, conforme descrição detalhada nas Tabelas 2 e 3 . Vale ressaltar que $29,1 \%$ dos alimentos tradicionais $(\mathrm{n}=629)$ e $8,3 \%$ dos diet, light e zero $(\mathrm{n}=3)$ são de sabor salgado, e $30 \%$ $(\mathrm{n}=189)$ e $66,7 \%(\mathrm{n}=2)$ deles apresentavam o açúcar nas três primeiras posições da lista de ingredientes, respectivamente. Dentre os alimentos tradicionais de sabor doce, $94,5 \%$ deles apresentaram o açúcar nas 3 primeiras posições da lista de ingredientes e nos diet, light e zero de sabor doce, 78,8\%. Quando analisados conjuntamente (tradicionais e diet, light e zero), $88,5 \%$ dos alimentos de sabor doce e $30,2 \%$ dos alimentos de sabor salgado possuem o açúcar nas três primeiras posições da lista de ingredientes.

Das 257 indústrias responsáveis pela produção dos alimentos analisados, 156 foram contatadas e apenas $12(7,7 \%)$ disponibilizaram as informações solicitadas referentes à quantidade de açúcar nas porções dos alimentos industrializados. Das 144 (92,3\%) restantes, 63 (43,8\%) relataram que tais informações são segredos de formulação do produto e, de acordo com a política dessas empresas, não poderiam fornecê-las; $32(22,2 \%)$ responderam que, como a legislação não obriga a rotulagem de açúcares, não há análise desses componentes separadamente; e 49 $(34,0 \%)$ não responderam ao e-mail.

\section{Discussão}

O estudo mostrou que na maioria dos alimentos industrializados da amostra analisada não há declaração da quantidade de açúcar na tabela nutricional de seus rótulos. No entanto, em grande parte deles, o açúcar aparece nas primeiras posições da lista de ingredientes, ou seja, é um ingrediente predominante na composição desses produtos, tanto nos tradicionais, quanto nos diet, light e zero. Aproximadamente 30\% dos alimentos que contém açúcar são de sabor salgado, e por isso a presença do açúcar não é evidente ao consumidor. Além da indústria de alimentos não disponibilizar a informação sobre a quantidade de açúcar nos rótulos de seus produtos e incluir o açúcar como principal ingrediente na maioria dos produtos alimentícios que o utilizam, quando solicitado via SAC, a informação sobre a quantidade de açúcar na maioria das vezes não é repassada.

A Pesquisa de Orçamentos Familiares (20082009) mostrou que $61,3 \%$ da população brasileira apresenta ingestão excessiva de açúcares, provenientes da adição de açúcar nos alimentos e do consumo de alimentos processados e ultraprocessados $^{19}$. Estima-se que, no Brasil, 21,5\% dos alimentos consumidos pela população brasileira é proveniente de alimentos ultraprocessados, ricos em açúcares, gorduras e sódio ${ }^{20}$. A associação do alto consumo de ultraprocessados à baixa disponibilidade de informação sobre açúcar, identificada no presente estudo, pode indicar que a população esteja consumindo altas quantidades de açúcares, de modo inconsciente. Isso mostra 
Tabela 1. Distribuição absoluta e percentual da declaração da quantidade de açúcar nos rótulos dos alimentos tradicionais e diet, light ou zero por categoria alimentar.

\begin{tabular}{|c|c|c|c|c|c|}
\hline \multirow[b]{2}{*}{ Categorias } & \multirow{2}{*}{$\begin{array}{c}\text { Número } \\
\text { total de } \\
\text { alimentos } \\
\text { analisados }\end{array}$} & \multicolumn{2}{|c|}{ Alimentos Tradicionais } & \multicolumn{2}{|c|}{$\begin{array}{c}\text { Alimentos Diet/Light/ } \\
\text { Zero }\end{array}$} \\
\hline & & $\begin{array}{c}\text { Total } \\
\text { Avaliado } \$\end{array}$ & $\begin{array}{c}\text { Total } \\
\text { Declarado } \\
\text { n }(\%)\end{array}$ & $\begin{array}{c}\text { Total } \\
\text { Avaliado } \$\end{array}$ & $\begin{array}{c}\text { Total } \\
\text { Declarado } \mathfrak{E} \\
\text { n }(\%)\end{array}$ \\
\hline 1. Açúcar e Mel & 61 & 61 & $0(0,0)$ & 0 & $0(0,0)$ \\
\hline 2. Balas, doces, bombons e confeitos & 201 & 201 & $71(35,3)$ & 0 & $0(0,0)$ \\
\hline 3. Bebidas & 259 & 259 & $35(13,5)$ & 0 & $0(0,0)$ \\
\hline 4. Carnes e Produtos Cárneos & 117 & 117 & $0(0,0)$ & 0 & $0(0,0)$ \\
\hline 5. Cereais e/ou produtos a base de cereais & 155 & 144 & $33(22,9)$ & 11 & $0(0,0)$ \\
\hline 6. Gelados Comestíveis & 80 & 80 & $5(6,2)$ & 0 & $0(0,0)$ \\
\hline 7. Molhos e Condimentos & 189 & 188 & $16(8,5)$ & 1 & $0(0,0)$ \\
\hline 8. Petiscos & 44 & 44 & $9(20,4)$ & 0 & $0(0,0)$ \\
\hline 9. Preparações Culinárias Industriais & 120 & 120 & $0(0,0)$ & 0 & $0(0,0)$ \\
\hline 10. Preparados para adicionar ao leite & 38 & 34 & $9(26,5)$ & 4 & $2(50,0)$ \\
\hline 11. Produtos de panificação e Biscoitos & 459 & 449 & $89(19,8)$ & 10 & $3(30,0)$ \\
\hline 12. Produtos Proteicos e Leveduras & 192 & 182 & $6(3,3)$ & 10 & $0(0,0)$ \\
\hline 13. Sobremesas e/ou pós para sobremesa & 230 & 230 & $29(12,6)$ & 0 & $0(0,0)$ \\
\hline 14. Sopas e Caldos & 50 & 50 & $9(18,0)$ & 0 & $0(0,0)$ \\
\hline 15. Suplementos Nutricionais & 5 & 5 & $0(0,0)$ & 0 & $0(0,0)$ \\
\hline Total & 2200 & 2164 & $311(14,4)$ & 36 & $5(13,9)$ \\
\hline
\end{tabular}

§Total Avaliado: Número total de alimentos industrializados tradicionais e diet/light/zero avaliados nesse estudo. £Total Declarado: Número total e porcentagem de alimentos industrializados tradicionais e diet/light/zero que declaravam a quantidade de açúcar na tabela nutricional dos rótulos alimentares.

a importância da regulamentação da declaração obrigatória da quantidade de açúcares adicionados no rótulo de alimentos industrializados.

No Brasil, esforços têm sido feitos no sentido de orientar sobre as consequências relacionadas ao alto consumo de alimentos processados e ultraprocessados ${ }^{21}$ e também para regulamentar a oferta de açúcares em alimentos industrializados por meio de um acordo entre o Ministério da Saúde e o setor produtivo ${ }^{22}$. Outra tentativa foi a de regulamentar a oferta, propaganda e venda de alimentos ricos em açúcares com a exigência da declaração do aumento do risco de obesidade e de outras doenças crônicas quando o açúcar for consumido em grande quantidade ${ }^{23}$. Em alguns países como o Chile e Colômbia, desde 2012, uma lei determina que os alimentos com alto teor de açúcares não possam ser oferecidos ou comercializados em estabelecimentos de educação infantil, fundamental e média ${ }^{24}$.

O açúcar é utilizado pela indústria de alimentos para melhor a palatabilidade dos produtos atraindo o consumidor, e também como aditivo alimentar, com diferentes funções: aromatizante, antioxidante, corante, preservativo, emulsificante, edulcorante, umectante, intensificador de sa- bor, enzima e nutrientes ${ }^{22}$. Isso pode justificar a presença do açúcar como principal ingrediente, tanto em alimentos doces como salgados.

Além de uma alta porcentagem de alimentos sem a declaração da quantidade de açúcar nos rótulos, não obtivemos resposta em relação a essa informação pela maioria das indústrias (92,3\%). Apesar da legislação brasileira que regulamenta a informação nutricional em rótulos de alimentos não prever a obrigatoriedade da informação quantitativa sobre açúcares ${ }^{14}$, o Código de Defesa do Consumidor, institui que "a informação adequada e clara sobre produtos e serviços, com especificação correta de quantidade, características, composição, qualidade e preço, bem como sobre os riscos que apresentem" é um direito básico do consumidor $^{25}$.

Com a ausência da informação sobre a quantidade de açúcar presente nos alimentos industrializados também não é possível calcular a quantidade real de açúcar consumida pelas pessoas. Isso prejudica o seguimento das recomendações nutricionais vigentes, que estabelece que o consumo de açúcar de crianças e adultos seja de até $10 \%$ do valor energético total (VET) da dieta, e que a redução para até $5 \%$ seria condicio- 
Tabela 2. Distribuição absoluta e percentual de alimentos tradicionais com o açúcar nas três primeiras posições da lista de ingredientes.

\begin{tabular}{|c|c|c|c|c|c|}
\hline \multirow{3}{*}{ Alimentos tradicionais } & \multirow{2}{*}{$\begin{array}{c}\text { Total de } \\
\text { alimentos } \\
\text { avaliados }\end{array}$} & \multicolumn{4}{|c|}{ Posição do açúcar na lista de ingredientes } \\
\hline & & $1^{\circ}$ & $2^{\circ}$ & $3^{\circ}$ & Total $^{*}$ \\
\hline & $\mathbf{n}$ & n (\%) & n (\%) & n (\%) & n (\%) \\
\hline 1. Açúcar e mel & 61 & $36(59,0)$ & $24(39,3)$ & $1(1,6)$ & $61(100,0)$ \\
\hline Açúcares & 15 & $15(100,0)$ & 0 & 0 & $15(100,0)$ \\
\hline Geleias & 46 & $21(45,6)$ & $24(52,2)$ & $1(2,2)$ & $46(100,0)$ \\
\hline 2. Balas, Doces, Bombons e Confeitos & 201 & $142(70,6)$ & $40(19,9)$ & $18(9,0)$ & $200(99,5)$ \\
\hline Balas, chicletes e pastilhas & 32 & $28(87,5)$ & $3(9,4)$ & $1(3,1)$ & $32(100,0)$ \\
\hline Bombons e chocolates & 75 & $70(93,3)$ & $4(5,3)$ & 0 & $74(98,6)$ \\
\hline Confeitos & 11 & $11(100,0)$ & 0 & 0 & $11(100,0)$ \\
\hline Doces & 83 & $33(39,7)$ & $33(39,7)$ & $17(20,5)$ & $83(100,0)$ \\
\hline 3. Bebidas & 259 & $55(21,2)$ & $141(54,4)$ & $50(19,3)$ & $246(94,9)$ \\
\hline Água de coco e água tônica & 3 & 0 & $2(66,7)$ & $1(33,3)$ & $3(100,0)$ \\
\hline Bebidas alcóolicas destiladas & 50 & $2(4,0)$ & $20(40,0)$ & $22(44,0)$ & $44(88,0)$ \\
\hline Bebidas alcóolicas fermentadas & 61 & $1(1,6)$ & $49(80,3)$ & $6(9,8)$ & $56(91,8)$ \\
\hline Chás & 2 & 0 & $2(100,0)$ & 0 & $2(100,0)$ \\
\hline Energéticos & 9 & 0 & $5(55,5)$ & $4(44,5)$ & $9(100,0)$ \\
\hline Pós para o preparo de capuccinos e café & & & & & \\
\hline leite & 16 & $13(81,3)$ & $3(18,7)$ & 0 & $16(100,0)$ \\
\hline Néctares de frutas & 30 & 0 & $14(46,7)$ & $14(46,7)$ & $28(93,4)$ \\
\hline Refrigerantes & 36 & $1(2,8)$ & $34(94,4)$ & $1(2,8)$ & $36(100,0)$ \\
\hline Sucos prontos para o consumo & 7 & 0 & $6(85,7)$ & $1(14,3)$ & $7(100,0)$ \\
\hline Sucos em pó & 36 & $35(97,2)$ & $1(2,8)$ & 0 & $36(100,0)$ \\
\hline Suplementos hidroeletrolíticos & 6 & 0 & $5(83,3)$ & $1(16,7)$ & $6(100,0)$ \\
\hline Xaropes (groselha, uva ou guaraná) & 3 & $3(100,0)$ & 0 & 0 & $3(100,0)$ \\
\hline 4. Carnes e produtos cárneos & 117 & $1(0,8)$ & $3(2,6)$ & $9(7,7)$ & $13(11,1)$ \\
\hline Almôndegas e hambúrgueres ${ }^{* *}$ & 5 & $1(20,0)$ & 0 & 0 & $1(20,0)$ \\
\hline Atuns e sardinhas em conserva ${ }^{\star *}$ & 6 & 0 & $1(16,7)$ & $2(33,3)$ & $3(50,0)$ \\
\hline Carnes temperadas ${ }^{\star *}$ & 33 & 0 & $1(3,0)$ & $2(6,0)$ & $3(9,0)$ \\
\hline Embutidos $^{\star *}$ & 73 & 0 & $1(1,4)$ & $5(6,8)$ & $6(8,2)$ \\
\hline 5. Cereais e/ou produtos a base de cereais & 144 & $23(16,0)$ & $72(50,0)$ & $18(12,5)$ & $113(78,5)$ \\
\hline Barras de cereais & 32 & $9(28,1)$ & $16(50,0)$ & $2(6,3)$ & $27(84,4)$ \\
\hline Barras de oleaginosas e frutas & 16 & $3(18,8)$ & $2(12,5)$ & $6(37,5)$ & $11(68,8)$ \\
\hline Cereais matinais & 41 & $6(14,6)$ & $28(68,3)$ & $3(7,3)$ & $37(90,2)$ \\
\hline Cereais para alimentação infantil & 15 & $1(6,7)$ & $12(80,0)$ & $2(13,3)$ & $15(100,0)$ \\
\hline Granolas & 13 & 0 & $9(69,2)$ & 0 & $9(69,2)$ \\
\hline Macarrão instantâneo ${ }^{* *}$ & 18 & $2(11,1)$ & $3(16,7)$ & $4(22,2)$ & $9(50,0)$ \\
\hline Massas (pizzas, pastel, panqueca) & 5 & 0 & 0,0 & $1(20,0)$ & $1(20,0)$ \\
\hline Outros & 4 & $2(50,0)$ & $2(50,0)$ & 0 & $4(100,0)$ \\
\hline 6. Gelados e comestíveis & 80 & $4(5,0)$ & $67(83,7)$ & $6(7,5)$ & $77(96,2)$ \\
\hline Açaís com guaraná & 3 & 0 & 0 & $3(100,0)$ & $3(100,0)$ \\
\hline Pós para o preparo de sorvetes & 4 & $4(100,0)$ & 0 & 0 & $4(100,0)$ \\
\hline
\end{tabular}

continua

nal, com possível aumento dos benefícios para a saúde ${ }^{9}$.

$\mathrm{O}$ fato de alguns alimentos salgados terem o açúcar nas 3 primeiras posições na lista de ingre- 
Tabela 2. Distribuição absoluta e percentual de alimentos tradicionais com o açúcar nas três primeiras posições da lista de ingredientes.

\begin{tabular}{|c|c|c|c|c|c|}
\hline \multirow{3}{*}{ Alimentos tradicionais } & \multirow{3}{*}{$\begin{array}{c}\text { Total de } \\
\text { alimentos } \\
\text { avaliados }\end{array}$} & \multicolumn{4}{|c|}{ Posição do açúcar na lista de ingredientes } \\
\hline & & $1^{\circ}$ & $2^{\circ}$ & $3^{\circ}$ & Total $^{*}$ \\
\hline & & n (\%) & n (\%) & n (\%) & n (\%) \\
\hline Sorvetes e sorbets & 73 & 0 & $67(91,8)$ & $3(4,1)$ & $70(95,9)$ \\
\hline 7. Molhos e condimentos & 188 & $7(3,7)$ & $41(21,8)$ & $34(18,1)$ & $82(43,6)$ \\
\hline Catchups $s^{\star *}$ & 4 & 0 & $4(100,0)$ & 0 & $4(100,0)$ \\
\hline Conservas $^{\star *}$ & 29 & 0 & $3(10,3)$ & $6(20,7)$ & $9(31,0)$ \\
\hline Extratos de tomate ${ }^{* *}$ & 9 & 0 & $8(88,9)$ & $1(11,1)$ & $9(100,0)$ \\
\hline Maioneses ${ }^{\star \star}$ & 8 & 0 & 0 & 0 & 0 \\
\hline Molhos e mistura para preparo de molhos ${ }^{\star \star}$ & 47 & $2(4,2)$ & $7(14,9)$ & $10(21,3)$ & $19(40,4)$ \\
\hline Molhos de tomate ${ }^{* *}$ & 54 & $1(1,8)$ & $7(13,0)$ & $14(25,9)$ & $22(40,7)$ \\
\hline Mostardas $^{* *}$ & 5 & 0 & $2(40,0)$ & 0 & $2(40,0)$ \\
\hline Pastas e patês ${ }^{\star *}$ & 10 & $3(30,0)$ & 0 & $1(10,0)$ & $4(40,0)$ \\
\hline Polpas de tomate ${ }^{\star *}$ & 6 & 0 & $5(83,3)$ & 0 & $5(83,3)$ \\
\hline Temperos prontos ${ }^{\star \star}$ & 16 & $1(6,3)$ & $5(31,2)$ & $2(12,5)$ & $8(50,0)$ \\
\hline 8. Petiscos (snacks) & 44 & 0 & $6(13,6)$ & $10(22,7)$ & $16(36,3)$ \\
\hline Batata frita ${ }^{\star *}$ & 7 & 0 & $1(14,3)$ & $3(42,8)$ & $4(57,1)$ \\
\hline Oleaginosas salgadas ${ }^{\star *}$ & 6 & 0 & 0 & $1(16,7)$ & $1(16,7)$ \\
\hline $\begin{array}{l}\text { Aperitivos a base de batatas, cereais, farinha ou } \\
\text { amido }^{\star \star}\end{array}$ & 31 & 0 & $5(16,1)$ & $6(19,4)$ & $11(35,5)$ \\
\hline 9. Preparações culinárias industriais & 120 & $3(2,5)$ & $11(9,2)$ & $10(8,3)$ & $24(20,0)$ \\
\hline Arroz temperado ${ }^{* *}$ & 6 & 0 & 0 & $2(33,3)$ & $2(33,3)$ \\
\hline Massas (lasanha, macarrão, nhoque) $)^{\star *}$ & 41 & 0 & 0 & 0 & 0 \\
\hline $\begin{array}{l}\text { Mistura para preparo (panqueca, purê e } \\
\text { risotos) }{ }^{* \star}\end{array}$ & 7 & 0 & $1(14,3)$ & 0 & $1(14,3)$ \\
\hline Papinhas doces & 6 & $1(16,7)$ & $1(16,7)$ & $4(66,7)$ & $6(100,0)$ \\
\hline Pizzas $^{\star *}$ & 32 & $1(3,1)$ & $3(9,4)$ & 0 & $4(12,5)$ \\
\hline Sanduiches ${ }^{* *}$ & 10 & 0 & $6(60,0)$ & $4(40,0)$ & $10(100,0)$ \\
\hline Outros (pão de alho, tortas, tortilhas, folhados...) ${ }^{* *}$ & 18 & $1(5,5)$ & 0 & 0 & $1(5,5)$ \\
\hline 10. Preparados para adicionar ao leite & 34 & $23(67,7)$ & $8(23,5)$ & $1(2,9)$ & $32(94,1)$ \\
\hline Achocolatados em pó & 11 & $11(100,0)$ & 0 & 0 & $11(100,0)$ \\
\hline Complementos nutricionais & 17 & $6(35,3)$ & $8(47,0)$ & $1(5,9)$ & $15(88,2)$ \\
\hline Pós para o preparo de bebidas (milk-shake) & 6 & $6(100,0)$ & 0 & 0 & $6(100,0)$ \\
\hline 11. Produtos de panificação e biscoitos & 449 & $104(23,2)$ & $230(51,2)$ & $51(11,3)$ & $385(85,7)$ \\
\hline Biscoitos doces (amanteigado, champagne) & 132 & $4(3,0)$ & $92(69,7)$ & $21(15,9)$ & $117(88,6)$ \\
\hline Biscoitos doces (recheado, wafer) & 88 & $40(45,5)$ & $48(54,5)$ & 0 & $88(100,0)$ \\
\hline Biscoitos salgados ${ }^{\star *}$ & 36 & 0 & $1(2,8)$ & $13(36,1)$ & $14(38,9)$ \\
\hline Bolos & 59 & $19(32,2)$ & $33(55,9)$ & $6(10,2)$ & $58(98,3)$ \\
\hline Misturas para preparo de bolos & 62 & $39(62,9)$ & $23(37,1)$ & 0 & $62(100,0)$ \\
\hline Pães e torradas ${ }^{\star *}$ & 55 & $1(1,8)$ & $23(41,8)$ & $10(18,2)$ & $34(61,8)$ \\
\hline Outros (tortas, panetones, rosca, waffle) & 17 & $1(5,9)$ & $10(58,8)$ & $1(5,9)$ & $12(70,6)$ \\
\hline
\end{tabular}

continua

lhos, salgadinhos e algumas preparações culinárias prontas para o consumo analisadas apresentavam o açúcar como um de seus primeiros ingredientes. A presença do açúcar em alimentos salgados pode ser justificada pelas funções tecnológicas associadas a ele $^{22}$.
Outro fator que pode levar a equívoco na escolha alimentar induzida pela falta de informação clara no rótulo é que a maioria das pessoas imagina que todos os alimentos diet, light e zero possuem um menor teor ou exclusão de açúcar, caloria e outros nutrientes. Quando trata-se de 
Tabela 2. Distribuição absoluta e percentual de alimentos tradicionais com o açúcar nas três primeiras posições da lista de ingredientes.

\begin{tabular}{|c|c|c|c|c|c|}
\hline \multirow{3}{*}{ Alimentos tradicionais } & \multirow{2}{*}{$\begin{array}{c}\text { Total de } \\
\text { alimentos } \\
\text { avaliados }\end{array}$} & \multicolumn{4}{|c|}{ Posição do açúcar na lista de ingredientes } \\
\hline & & $1^{\circ}$ & $2^{\circ}$ & $3^{\circ}$ & Total $^{*}$ \\
\hline & $\mathbf{n}$ & n (\%) & n (\%) & n (\%) & n (\%) \\
\hline 12. Produtos Protéicos e Leveduras & 182 & $3(1,6)$ & $95(52,2)$ & $65(35,7)$ & $163(89,5)$ \\
\hline Alimentos com soja & 20 & $1(5,0)$ & $6(30,0)$ & $12(60,0)$ & $19(95,0)$ \\
\hline Bebidas lácteas & 58 & 0 & $17(29,3)$ & $29(50,0)$ & $46(79,3)$ \\
\hline Iogurtes & 66 & 0 & $46(69,7)$ & $18(27,3)$ & $64(97,0)$ \\
\hline Leites fermentados & 25 & $1(4,0)$ & $17(68,0)$ & $4(16,0)$ & $22(88,0)$ \\
\hline Queijos** & 2 & $1(50,0)$ & 0 & 0 & $1(50,0)$ \\
\hline Queijos petit suisse & 11 & 0 & $9(81,8)$ & $2(18,2)$ & $11(100,0)$ \\
\hline 13. Sobremesas e pós para sobremesas & 230 & $109(47,4)$ & $86(37,4)$ & $27(11,7)$ & $222(96,5)$ \\
\hline Pós para o preparo de sobremesas & 101 & $98(97,0)$ & $3(3,0)$ & 0 & $101(100,0)$ \\
\hline Sobremesas lácteas & 15 & $2(13,3)$ & $6(40,0)$ & $3(20,0)$ & $11(73,3)$ \\
\hline Sobremesas prontas para consumo & 114 & $9(7,9)$ & $77(67,5)$ & $24(21,0)$ & $110(96,5)$ \\
\hline 14. Sopas e caldos & 50 & 0 & 0 & $1(2,0)$ & $1(2,0)$ \\
\hline Misturas para preparo de cremes e sopas ${ }^{\star \star}$ & 45 & 0 & 0 & $1(2,2)$ & $1(2,2)$ \\
\hline Sopas $* *$ & 5 & 0 & 0 & 0 & 0 \\
\hline 15. Suplementos nutricionais & 5 & $3(60,0)$ & $1(20,0)$ & $1(20,0)$ & $5(100,0)$ \\
\hline Barras de proteínas & 4 & $3(75,0)$ & $1(25,0)$ & 0 & $4(100,0)$ \\
\hline Suplemento vitamínico e mineral & 1 & 0 & 0 & $1(100,0)$ & $1(100,0)$ \\
\hline Total & 2164 & $513(23,7)$ & $825(38,1)$ & $302(14,0)$ & $1640(75,8)$ \\
\hline
\end{tabular}

*Soma dos alimentos que têm açúcar nas 3 primeiras posições da lista de ingredientes (\% em relação ao total de alimentos avaliados). ${ }^{*}$ Alimentos de sabor salgado.

Tabela 3. Distribuição absoluta e percentual de alimentos diet/light/zero com o açúcar nas três primeiras posições da lista de ingredientes.

\begin{tabular}{|c|c|c|c|c|c|}
\hline \multirow{3}{*}{ Alimentos diet/light/zero } & \multirow{2}{*}{$\begin{array}{c}\text { Total de } \\
\text { alimentos } \\
\text { avaliados }\end{array}$} & \multicolumn{4}{|c|}{ Posição do açúcar na lista de ingredientes } \\
\hline & & $1^{\circ}$ & $2^{\circ}$ & $3^{\circ}$ & Total $^{*}$ \\
\hline & $\mathbf{n}$ & n (\%) & n (\%) & n (\%) & n (\%) \\
\hline 5. Cereais e/ou produtos a base de cereais & 11 & 0 & $2(18,2)$ & $6(54,5)$ & $8(72,7)$ \\
\hline Barras de cereais & 7 & 0 & $1(14,3)$ & $3(42,8)$ & $4(57,1)$ \\
\hline Granolas & 4 & 0 & $1(25,0)$ & $3(75,0)$ & $4(100,0)$ \\
\hline 7. Molhos e condimentos & 1 & 0 & 0 & 0 & 0 \\
\hline Molhos de tomate ${ }^{* *}$ & 1 & 0 & 0 & 0 & 0 \\
\hline 10. Preparados para adicionar ao leite & 4 & $4(100,0)$ & 0 & 0 & $4(100,0)$ \\
\hline Achocolatados em pó & 4 & $4(100,0)$ & 0 & 0 & $4(100,0)$ \\
\hline 11. Produtos de panificação e biscoitos & 10 & $2(20,0)$ & $1(10,0)$ & $5(50,0)$ & $8(80,0)$ \\
\hline Biscoitos doces (amanteigado, champagne) & 7 & $2(28,6)$ & $1(14,3)$ & $3(42,8)$ & $6(85,7)$ \\
\hline Biscoitos salgados ${ }^{\star *}$ & 2 & 0 & 0 & $2(100,0)$ & $2(100,0)$ \\
\hline Waffle & 1 & 0 & 0 & 0 & 0 \\
\hline 12. Produtos Proteicos e Leveduras & 10 & $1(10,0)$ & $6(60,0)$ & $1(10,0)$ & $8(80,0)$ \\
\hline Alimentos com soja & 3 & $1(33,3)$ & $2(66,7)$ & 0 & $3(100,0)$ \\
\hline Bebidas lácteas & 1 & 0 & 0 & $1(100,0)$ & $1(100,0)$ \\
\hline Iogurtes & 5 & 0 & $3(100,0)$ & 0 & $3(100,0)$ \\
\hline Leites fermentados & 1 & 0 & $1(100,0)$ & 0 & $1(100,0)$ \\
\hline Total & 36 & $7(19,5)$ & $9(25,0)$ & $12(33,3)$ & $28(77,8)$ \\
\hline
\end{tabular}

${ }^{\star}$ Soma dos alimentos que têm açúcar nas 3 primeiras posições da lista de ingredientes (\% em relação ao total de alimentos avaliados). ${ }^{* *}$ Alimentos de sabor salgado. 
um alimento diet, light e zero a legislação somente obriga a declaração da informação do açúcar no rótulo destes quando não contém ou quando este não possui adição de açúcares ${ }^{18}$. Ou seja, em casos como os dos alimentos analisados, em que há a presença de açúcar, não existe a obrigatoriedade desta declaração nos rótulos, mesmo sendo produtos diet, light e zero ${ }^{15}$.

No entanto, estão em vigência atualmente dois Regulamentos Técnicos do Mercosul sobre Rotulagem Nutricional de alimentos embalados e Informação Nutricional Complementar. Eles se aplicam aos alimentos produzidos e comercializados na Argentina, Brasil, Uruguai e Paraguai, e determinam que se houver declaração de propriedades nutricionais relacionada à carboidratos (informação nutricional complementar, como por exemplo o alimento light relacionado à redução de açúcares), a quantidade de açúcar deve ser declarada na tabela nutricional ${ }^{26,27}$. Por outro lado, na União Europeia e Estados Unidos da América, a quantidade de açúcares presentes no alimento deve obrigatoriamente ser declarada nos rótulos de todos os alimentos industrializados ${ }^{28,29}$.

\section{Colaboradores}

CC Japur e FRO Penaforte participaram da concepção do trabalho. CC Japur e DCB Assunção participaram do planejamento e redação do trabalho. DCB Assunção e RAB Batista participaram da coleta e digitação dos dados. CC Japur, DCB Assunção, RAB Batista e FRO Penaforte participaram da análise, interpretação e revisão crítica do trabalho, e aprovaram a versão final encaminhada.
Os dados em relação à presença do açúcar em alimentos industrializados podem estar subestimados, uma vez que foram incluídos na análise apenas alimentos com a denominação sacarose e/ ou açúcar na lista de ingredientes, excluindo outros tipos de açúcares (mono e dissacarídeos), o que se configura como uma limitação do estudo.

\section{Conclusão}

A disponibilidade da informação sobre quantidade de açúcar em alimentos industrializados é precária, tanto na observação dos rótulos dos alimentos (em que apenas 15\% dos produtos declaram a quantidade de açúcar na porção do alimento), quanto por meio do SAC (em que 92\% das indústrias não repassam tal informação). Esses dados são preocupantes, uma vez que dentre os alimentos avaliados, $88,5 \%$ dos alimentos de sabor doce e $30,2 \%$ dos alimentos de sabor salgado possuem o açúcar nas três primeiras posições da lista de ingredientes, ou seja, têm o açúcar como principal ingrediente.

\section{Agradecimentos}

Ao Conselho Nacional de Desenvolvimento Científico e Tecnológico (CNPq), pelo auxílio ao projeto com a concessão de uma bolsa de estudos, e à gerência do hipermercado, que, gentilmente, autorizou a coleta de dados dentro de suas dependências. 


\section{Referências}

1. Maire B, Lioret S, Gartner A, Delpeuch F. Nutritional transition and non-communicable diet-related chronic diseases in developing countries. Sante 2002; 12(1):45-55.

2. Schmidt MI, Duncan BB, Silva GA, Menezes AM, Monteiro CA, Barreto SM, Chor D, Menezes PR. Chronic non-communicable diseases in Brazil: burden and current challenges. Lancet 2011; 377(9781):1949-1961.

3. Louzada MLC, Baraldi LG, Steele EM, Martins APB, Canella DS, Moubarac J-C, Levy RB, Cannon G, Afshin A, Imamura F, Mozaffarian D, Monteiro CA. Consumption of ultra-processed foods and obesity in Brazilian adolescents and adults. Prev Med 2015; 81:9-15.

4. Costa CS, Del-Ponte B, Assunção MCF, Santos IS. Consumption of ultra-processed foods and body fat during childhood and adolescence: a systematic review. Public Health Nutr 2018; 21(1):148-159.

5. Fiolet T, Srour B, Sellem L, Kesse-Guyot E, Allès B, Méjean C, Deschasaux M, Fassier P, Latino-Martel P, Beslay M, Hercberg S, Lavalette C, Monteiro CA, Julia C, Touvier M. Consumption of ultra-processed foods and cancer risk: results from NutriNet-Santé prospective cohort. BMJ 2018; 360:k322.

6. Rauber F, Louzada MLC, Steele E, Millett C, Monteiro CA, Levy RB. Ultra-Processed Food Consumption and Chronic Non-Communicable Diseases-Related Dietary Nutrient Profile in the UK (2008-2014). Nutrients 2018; 10(5):587.

7. Basu S, McKee M, Galea G, Stuckler D. Relationship of soft drink consumption to global overweight, obesity, and diabetes: a cross-national analysis of 75 countries. Am J Public Health 2013; 103(11):2071-2077.

8. Siervo M, Montagnese C, Mathers JC, Soroka KR, Stephan BC, Wells JC. Sugar consumption and global prevalence of obesity and hypertension: an ecological analysis. Public Health Nutr 2014; 17(3):587-596.

9. World Health Organization (WHO). Guideline: Sugars intake for adults and children. Genebra: WHO; 2015.

10. Scapin T, Fernandes AC, Proença RPC. Added sugars: Definitions, classifications, metabolism and health implications. Rev Nutr 2017; 30(5):663-677.

11. Vos MB, Kaar JL, Welsh JA, Van Horn LV, Feig DI, Anderson CAM, Patel MJ, Munos JC, Krebs NF, Xanthakos SA, Johnson RK, American Heart Association Nutrition Committee of the Council on Lifestyle and Cardiometabolic Health, Council on Clinical Cardiology, Council on Cardiovascular Disease in the Young, Council on Cardiovascular and Stroke Nursing, Council on Epidemiology and Prevention, Council on Functional Genomics and Translational Biology, and Council on Hypertension. Added Sugars and Cardiovascular Disease Risk in Children: A Scientific Statement From the American Heart Association. Circulation 2017; 135(19):e1017-e1034.

12. Institute of Medicine. Dietary Reference Intakes for Energy, Carbohydrate, Fiber, Fat, Fatty Acids, Cholesterol, Protein, and Amino Acids (Macronutrients) Washington: National Academies Press; 2005.
13. Organização Pan Americana de Saúde (OPAS). Modelo de Perfil Nutricional da Organização Pan-Americana da Saúde. Brasília: OPAS; 2016.

14. Brasil. Secretaria de Vigilância Sanitária (Anvisa). Resolução RDC n 360, de 23 de dezembro de 2003 Regulamento técnico sobre rotulagem de alimentos embalados. Diário Oficial da União 2003; $23 \mathrm{dez}$.

15. Brasil. Secretaria de Vigilância Sanitária (Anvisa). Portaria ${ }^{\circ} 29$, de 13 de janeiro de 1998. Regulamento técnico para fixação de identidade e qualidade de alimentos para fins especiais. Diário Oficial da União 1998; 29 jan.

16. Associação Brasileira de Supermercados (ABRAS). Ranking ABRAS/SuperHiper. São Paulo: ABRAS; 2014

17. Brasil. Secretaria de Vigilância Sanitária (Anvisa). Guia de Procedimentos para Pedidos de Inclusão e Extensão de Uso de Aditivos Alimentares e Coadjuvantes de Tecnologia de Fabricação na Legislação Brasileira. Brasília: Anvisa; 2015.

18. Brasil. Secretaria de Vigilância Sanitária (Anvisa). Resolução RDC no 54 de 12 de novembro de 2012 Regulamento técnico sobre informação nutricional complementar. Diário Oficial da União 2012; 12 nov.

19. Brasil. Instituto Brasileiro de Geografia e Estatística (IBGE). Coordenação de Trabalho e Rendimento. Pesquisa de orçamentos familiares 2008-2009: análise do consumo alimentar pessoal no Brasil. Rio de Janeiro: IBGE; 2011.

20. Louzada MLC, Martins APB, Canella DS, Baraldi LG, Levy RB, Claro RM, Moubarac J-C, Cannon G, Monteiro CA. Ultra-processed foods and the nutritional dietary profile in Brazil. Rev Saude Publica 2015; 49:38.

21. Brasil. Ministério da Saúde (MS). Guia Alimentar para a População Brasileira. Brasília: MS; 2014.

22. Brasil. Ministério da Saúde (MS). Plano de Redução de açúcar em alimentos industrializados. Brasília: MS; 2017.

23. Brasil. Secretaria de Vigilância Sanitária (Anvisa). Resolução RDC n 24, de 15 de junho de 2010. Dispõe sobre a oferta, propaganda, publicidade, informação e outras práticas correlatas cujo objetivo seja a divulgação e a promoção comercial de alimentos considerados com quantidades elevadas de açúcar, de gordura saturada, de gordura trans, de sódio, e de bebidas com baixo teor nutricional. Diário Oficial da União 2010; 24 jun.

24. Fraser B. Latin American countries crack down on junk food. Lancet 2013; 382(9890):385-386.

25. Brasil. Lei no 8078, de 11 de setembro de 1990. Dispõe sobre a proteção do consumidor e dá outras providências. Diário Oficial da União 1990; 11 set.

26. Mercado Comum do Sul (MERCOSUL). MERCOSUL/GMC/RES n ${ }^{\circ}$ 46/03. Regulamento Técnico Mercosul sobre Rotulagem Nutricional de Alimentos Embalados. Montevidéu: Mercosul; 2003.

27. Mercado Comum do Sul (MERCOSUL). MERCOSUL/GMC/RES. $n^{\circ}$ 01/12. Regulamento Técnico Mercosul sobre Informação Nutricional Complementar (Declarações de Propriedades Nutricionais). Montevidéu: Mercosul; 2012. 
28. Food and Drug Administration (FDA). Federal Register - Food Labeling: Revision of the Nutrition and Supplement Facts Labels. Silver Spring: FDA; 2016.

29. European Parliament. Regulation (EU) $n^{\circ} 1169 / 2011$ of the European Parliament and of the Council of 25 October 2011 on the provision of food information to consumers. EU; 2011.

Artigo apresentado em 22/03/2019

Aprovado em 05/06/2019

Versão final apresentada em 07/06/2019

Editores-chefes: Romeu Gomes, Antônio Augusto Moura da Silva 